\title{
Validação da Escala de Crenças sobre Abuso Sexual (ECAS) no Contexto Brasileiro
}

\author{
Camila de Alencar Pereira ${ }^{1}$ \\ Silvana Carneiro Maciel $^{2}$ \\ Camila Cristina Vasconcelos Dias ${ }^{2}$ \\ Tátia Mirellis de Oliveira Alexandre ${ }^{3}$ \\ Marcelo Xavier de Oliveira ${ }^{2}$ \\ Carlos Eduardo Pimentel ${ }^{2}$ \\ ${ }^{1}$ Faculdade Maurício de Nassau, João Pessoa, PB \\ ${ }^{2}$ Universidade Federal da Paraíba, João Pessoa, PB \\ ${ }^{3}$ Casa de acolhimento para crianças e adolescentes da cidade de Patos, Patos, PB
}

\begin{abstract}
Resumo
O presente artigo objetiva adaptar e validar para o contexto brasileiro a Escala de Crenças sobre Abuso Sexual (ECAS) de origem portuguesa, composta por 17 itens e três fatores. Para tanto foram realizados dois estudos, no primeiro foi feita uma análise fatorial exploratória com amostra de 208 estudantes universitários, obtendo-se uma estrutura unifatorial, divergindo da escala original, com alfa de Cronbach de 0,88. Diante da discordância, foi realizado um segundo estudo, visando confirmar a estrutura fatorial da escala, com amostra de 209 estudantes universitários. Portanto, o resultado confirmou como melhor modelo a estrutura unifatorial da escala, com redução de 17 para 11 itens, obtendo-se alfa de Cronbach de 0,84. Sendo assim, a adaptação e validação da ECAS contribui para preencher uma carência existente no Brasil em relação a instrumentos voltados a mensurar as crenças sobre o abuso sexual infantojuvenil, podendo colaborar com estudos e intervenções na área.

Palavras-chave: crenças, abuso sexual, crianças, adolescentes, escala
\end{abstract}

\section{Validation of the Sexual Abuse Beliefs Scale (SABS) in the Brazilian Context}

\begin{abstract}
This article aims to adapt and validate for the Brazilian context the Sexual Abuse Beliefs Scale (SABS), from a Portuguese origin, composed by 17 items and 3 factors. For this, two studies were realized. In the first, an exploratory factorial analysis was performed with a sample of 208 undergraduate students, obtaining a unifactorial structure, differing form the original scale, with Cronbach's alpha of 0.88 . Facing this disagreement, a second study was realized to confirm the factorial structure of the scale, with 209 undergraduate students. Therefore, the result confirmed the unifactorial structure as the best model for the scale, with reduction from 17 to 11 items, obtaining Cronbach's alpha of 0.84 . Thus the adaptation and validation of SABS contributes to fill a gap existing in Brazil regarding to instruments aimed at measuring beliefs about child sexual abuse, and can collaborate with studies and interventions in the area.
\end{abstract}

Keywords: belief; sexual abuse; children; teenagers; scale.

Validación de la Escala de Creencias del Abuso Sexual (ECAS) en el contexto Brasileño

\section{Resumen}

En este artículo tiene por objetivo adaptar y validar el contexto brasileño la Escala de Creencias sobre Abuso Sexual (ECAS), de origen portugués, formada por 17 ítems y 3 factores. Para ello, fueron realizados dos estúdios, en el primero fue hecho um análisis factorial exploratória com muestra 208 estudiantes universitários, alcanzando uma estructura unifactorial, divergièndose de al escala original com Alfa de Crobach de 0,88. Ante la discrepância, fue realizado um segundo estudio, visando confirmar la estructura factorial de la escala, con muestra de 209 estudiantes universitários. Por lo tanto, el resultado pudo confirmar como mejor modelo la estrucutura unifactorial de la escala, com reducción de 17 para 11 ítems, obteniéndose Alfa de Cronbach de 0,84. Así siendo, la adaptación y validación de la ECAS contribuye para llenar uma carência existente en Brasil con relación a instrumentos direcionados a mensurar las crencias acerca del abuso sexual infantojuvenil, pudiendo colaborar com estúdios e intervenciones em el área.

Palabras-clave: creencias; abuso Sexual; niños; adolescentes; escala.

\section{Introdução}

A violência se revela um grave problema social, na medida em que afeta substancial e prejudicialmente as relações existentes dentro de uma sociedade, ameaçando a paz objetivada pelo agrupamento humano e configura, segundo Garbin, Dias, Rovida e Garbin (2015), um grande desafio mundial. A violência atinge 
a população nas suas mais variadas formas e cenários, perpassando todas as fases de desenvolvimento humano.

No cenário da violência, inclusive da sexual, dentre as vítimas mais corriqueiras, destacam-se as crianças e os adolescentes por serem mais vulneráveis tendo em vista, sobretudo, a fragilidade e dependência inerentes aos seus estágios de desenvolvimento físico e mental. No que se refere à violência sexual, esta pode ser dividida em abuso sexual (AS), que compreende atos e/ ou atividades cujo objetivo é a obtenção de satisfação lascívia do agressor (Florentino, 2015; Freire \& Alberto, 2013; Lima, Alberto, Santos, Brito, \& Silva, 2014; Pincolini \& Hutz, 2014; Sá et al., 2016); e Exploração Sexual (ES) a qual consiste em práticas relacionadas à comercialização, em que se paga à vítima ou a terceiros (Pincolini \& Hutz, 2014).

$\mathrm{Na}$ legislação brasileira, Lei no ${ }^{\circ} 13.431 / 2017$ no seu art. $4^{\circ}$, III, violência sexual é definida como toda ação que intimide a criança ou o adolescente a cometer ou testemunhar conjunção carnal ou qualquer outra conduta libidinosa, incluindo exibição do corpo em mídias por meio eletrônico ou não; havendo ou não conjunção carnal. Por sua vez, a exploração comercial é definida como a troca da atividade sexual por remuneração ou qualquer outra forma de compensação.

Verifica-se, então, que o abuso sexual é entendido como uma violência que transcende o mero ato sexual, e que pode ser articulado a outras espécies de violência como a física, a emocional e a negligencial (Maria, 2012). Mesmo sabendo da gravidade tanto do abuso quanto da exploração comercial, optou-se, neste trabalho, por aprofundar apenas as questões envolvendo o abuso sexual voltado para infantojuvenil (ASI), por este ser considerado um dos maiores problemas sociais e de saúde pública da contemporaneidade (Organização Mundial de Saúde [OMS], 2014).

Os dados de prevalência sobre abuso sexual contra crianças e adolescentes se revelam aquém da realidade, haja vista que muitas ocorrências não são reconhecidas, diagnosticadas, notificadas e/ou denunciadas (Nicoletti, Giacomozzi, \& Cabral, 2017). Apesar dessa circunstância, a OMS (2016) informou que uma a cada cinco mulheres e um a cada 13 homens foram vítimas de abuso sexual durante a infância. Dessa forma, cabe destacar que mesmo em um nicho considerado pequeno, em virtude do baixo número de casos registrados, é alto o índice de vítimas desse tipo de violência.

Uma estimativa global sobre a prevalência do abuso sexual infantil foi realizada por Pereda, Guilera,
Forns, \& Gómez-Benito (2009) por meio de um estudo de metanálise de 65 artigos de 22 países, observando que a média de prevalência de abuso sexual é maior no que diz respeito ao sexo feminino (19,7\%). Outro estudo de meta-análise realizado com 217 publicações entre os anos 1980 e 2008, constatou um indicativo acentuado por gênero de vítimas de abuso sexual, sendo 18\% do sexo feminino (Stoltenborgh, IJzendoorn, Euser, \& Bakerman-Kranenburg, 2011). Observou-se, por meio dos estudos citados, que a prevalência de abuso sexual contra crianças e adolescentes é maior no que diz respeito ao sexo feminino.

O Programa Nacional de Enfrentamento da Violência Sexual contra Crianças e Adolescentes realizado no Brasil divulgou o relatório referente a notificações de abuso sexual no de 2011, constatando que, das denúncias recebidas pelo disque $100,77 \%$ das vítimas eram meninas e $23 \%$ eram meninos. Nesse mesmo relatório, foi apontado que das violências sexuais denunciadas $60 \%$ foram referentes ao abuso sexual, sendo o restante sobre exploração sexual, pornografia e tráfico de crianças e adolescentes. De acordo com Santana, Santana e Lopes (2011), estima-se que, a cada hora, sete crianças são sexualmente abusadas ou que, por dia, 165 crianças sejam vítimas de abuso sexual.

Justamente pela característica de grave violência com repercussão de grande intensidade, a prática de abuso sexual contra crianças e adolescentes recebe, na legislação brasileira, um tratamento especial nos mais variados campos do direito. Há o destaque para a Lei $\mathrm{n}^{\circ}$ 8.069/1990, que estabelece o Estatuto da Criança e do Adolescente (ECA) e a Lei n ${ }^{\circ} 13.431 / 2107$, que, além de modificar alguns dispositivos do ECA, institui o sistema de garantia de direitos da criança e do adolescente vítimas ou testemunhas de violência. Há, assim, medidas legais de caráter preventivo, quando se objetiva nitidamente a proteção infantojuvenil - a exemplo da capacitação de profissionais para identificação de casos de violência, prevista no art. 70-A, III, do ECA -, bem como de caráter repressivo, notadamente na política criminal de adoção de penas mais severas para os agentes que cometem esse tipo de delito, enquadrando-o como crime hediondo.

Ressalta-se, pois, que o abuso sexual é um evento complexo que deixa marcas que vão além dos aspectos físicos e atingem, também, a dinâmica social, bem como o funcionamento psíquico da vítima, refletindo de maneira plena na saúde biopsicossocial (Silva, Ferriani, Beserra, Roque, \& Carlos, 2013). As repercussões são diversas e podem ser expressas em sentimentos 
de raiva, culpa, vergonha, medo e desamparo, sendo oriundo dessa vivência o desenvolvimento de sintomas como depressão, queixas somáticas, transtornos de ansiedade, alimentares e dissociativos, déficit de atenção, baixa autoestima, baixo rendimento escolar e transtorno de estresse pós-traumático, conduta hipersexualizada, abuso de substâncias, fugas do lar, furtos, isolamento social, agressividade, mudanças nos padrões de sono, comportamentos autodestrutivos, dentre outros (Espindola \& Batista, 2013; Florentino, 2015; Von Hohendorff; Koller, \& Habigzang, 2015; Platt, Back, Hauschild, \& Guedert, 2018; Soares et al., 2016).

Algumas formas de enfrentamento do problema vêm sendo desenvolvidas, como é o caso de programas de prevenção ao abuso sexual. Sobre o desenvolvimento de tais programas, Maria (2012) destaca que a respectiva avaliação exige a utilização de medidas aproximadas que possam traçar um panorama de suas eficácias, associadas às mudanças ao nível dos conhecimentos, das atitudes e das crenças que estão relacionadas aos comportamentos a que os programas visam combater.

No entanto, apesar dos vários estudos na área do abuso sexual infantojuvenil, da existência de leis e da presença de inúmeros movimentos sociais em prol da proteção das crianças no que se refere à violência sexual, pode-se ressaltar que ainda existem muitas lacunas sobre o tema. Essas omissões acabam por comprometer a ampla discussão social e o surgimento de trabalhos preventivos e de formas mais adequadas de lidar com o fenômeno.

No que se refere às crenças, estas podem ser definidas como uma representação aceita por uma ou mais pessoas, atuando de forma direta nas atitudes e comportamentos humanos, agindo na socialização e formação cultural e ideológica da sociedade (Salgado, 2012). Para Camino, Torres, Lima e Pereira (2013), as crenças orientam as condutas sociais e influenciam as tomadas de decisões. Diante disso, analisar as crenças sobre a natureza do abuso sexual contra crianças e adolescentes é primordial, posto que, de acordo com Maciel, Pereira, Lima e Souza (2015), as crenças sobre grupos sociais funcionam como princípios organizadores que motivam os comportamentos e as atitudes. Dentro desse contexto foram pesquisados instrumentos destinados a mensurar as crenças sobre o abuso sexual infantojuvenil, de forma a contribuir para o desenvolvimento de pesquisas na área no contexto brasileiro.

Mesmo não pretendendo esgotar a temática, procurou-se encontrar uma escala compatível com o objetivo de investigar as crenças acerca do abuso sexual infantojuvenil. Para tanto, foi realizada uma pesquisa na literatura, nas bases Google Acadêmico no período de setembro a novembro de 2016 - filtrando-se a análise a partir dos termos vinculados "escala-crenças-abuso sexual", "escala-violência sexual", adicionando em ambas os termos "criança", "adolescente" e "infantojuvenil". Foram utilizados os termos em português e em inglês, não se delimitando, porém, o período de busca. Alguns instrumentos voltados para as temáticas pesquisadas foram encontrados, como: Escala de Crenças sobre Violência Sexual (ECVS) (Martins, Machado, Abrunhosa, \& Manita, 2012), Escala Infantil de Sintomas del Trastorno de Estrés Postraumático (CPSS) (Bustos, Rincón, \& Aedo, 2009), Questionário para Avaliação de Comportamento, Sintomas Físicos e Emocionais de Crianças de 2 a 12 Anos de Idade (Salvagni \& Wagner, 2006), Index of Spouse Abuse (Santos-Iglesias, Sierra, \& Vallejo-Medina, 2013), CSBI - Child Sexuality Behavior Inventory (Rossetti, 2012) e Illinois Rape Myth Acceptance Scale, IRMA (Scarpati, Guerra, \& Duarte, 2014).

Observou-se que muitos dos instrumentos encontrados enfocavam a mensuração das consequências comportamentais e emocionais das vítimas e não se propunham a identificar as crenças dos indivíduos sobre o abuso sexual infantojuvenil. Dentre as escalas encontradas, apenas a Escala de Crenças sobre Violência Sexual (ECVS) trabalhava com a temática das crenças e da violência sexual só que de forma geral, abordando todos os tipos de violência sexual e em todas as faixas etárias e não apenas a infantojuvenil, fugindo, assim, da temática proposta pelo presente estudo.

Dentre os estudos, foi encontrada a Escala de Crenças sobre Abuso Sexual (ECAS) elaborada por Machado, Gonçalves e Matos (2000), e validada para Portugal. Esta incluiu assertivas voltadas para as crenças em torno dos abusos sexuais especificamente cometidos contra crianças e adolescentes, sendo, assim, o instrumento que mais se adequa, dentre os pesquisados, aos objetivos traçados para este estudo.

Essa escala foi criada a partir da observação clínica de crianças e adolescentes que sofreram algum tipo de abuso sexual e da literatura especializada voltada aos mitos e às crenças culturais existentes em relação a essa temática. Os autores utilizaram, também, como fonte de dados para construção desse instrumento os relatos obtidos com a observação clínica de abusadores, além de estudos que apontam para a existência de certas estratégias cognitivas legitimadoras desse tipo de comportamento sexual fora do padrão, a exemplo da legitimação do abuso pela 
sedução infantil e da legitimação do abuso pelo perfil do abusador (Ladeiro, 2014).

O instrumento original possui 17 itens dividido em três fatores: legitimação do abuso pela sedução infantil; legitimação pela ausência de violência; e a crença de que o abuso não existe quando o ofensor não corresponde a um estereótipo pré-definido (Ladeiro, 2014). Dessa forma os autores propuseram mensurar crenças referentes ao perfil da vítima (por exemplo: item 2 - Só as meninas são vítimas de abuso sexual); perfil do abusador (por exemplo: item 5 - Os abusadores são pessoas que parecem diferentes das pessoas normais), além da crença da existência do abuso associado à presença de violência física (por exemplo: item 7 - Só se pode falar em abuso quando há violência).

A partir desses objetivos de mensuração da escala, foi possível constatar uma correspondência com a literatura. O estudo de Shackel (2008) aponta que existe a representação do abuso sexual infantojuvenil mediante o uso da violência e da força física, fato este corroborado por Santos (2011), que, em estudo com estudantes universitários e o estímulo abuso sexual de crianças e adolescentes, encontrou o termo "violência" como um dos mais frequentes.

No que se refere às crenças sobre o perfil da vítima, esta é vista como uma criança sedutora, o que acaba por desresponsabilizar o agressor, promovendo a culpa na vítima. Nessa perspectiva, Eloy (2010) afirma que a erotização precoce da criança incentivada pelas mídias e condutas sociais atuais acaba por despertar a representação social do fim da infância nos profissionais que atuam nesse cenário, o que pode ocasionar uma modificação na forma como o fenômeno é visto. Já no que diz respeito ao perfil do abusador, Fazenda (2010) destaca a crença de que o abusador sexual possui boa integração e imagem social, não sendo possível o reconhecimento prévio do agressor diante de características pessoais ou estereótipos predeterminados.

$\mathrm{O}$ instrumento em sua totalidade revela, ainda, uma medida geral de tolerância e legitimação do abuso sexual, em que os totais de cada fator se referem às crenças centrais e latentes a essa legitimação (Ladeiro, 2014). Essa escala foi usada em outras pesquisas no contexto português, a exemplo das realizadas por Fazenda (2010), que a aplicou em uma amostra de profissionais da saúde, mais especificamente em médicos e enfermeiros, e por Querido (2010), que utilizou em uma amostra de educadores infantis, que demonstraram coeficientes de consistência interna, alfa de Cronbach, de 0,80 e 0,84 , respectivamente.
Diante da necessidade de se trabalhar com a temática das crenças sobre o abuso sexual infantojuvenil, e da carência de instrumentos na área, o presente estudo teve como objetivo adaptar e validar para o contexto brasileiro a Escala de Crenças sobre Abuso Sexual (ECAS). Compreendendo que a escala possui pontos fortes como a questão da elaboração dos itens no que diz respeito às principais crenças acerca do abuso sexual infantojuvenil encontradas na literatura - das crenças sobre o perfil do abusado, fantasias da criança e criança sedutora (Almeida, 2003) - e pelo fato de constituir uma medida genérica de tolerância/legitimação do abuso sexual; mas possui fraqueza enquanto divisão dos fatores onde alguns itens possuem duplicidades nos fatores a exemplo do item 4 descrito nos fatores legitimação do abuso pela sedução infantil e crença de que o abuso não existe quando o ofensor não corresponde a um estereótipo predefinido. Já os itens 09,11 e 13 estão inseridos nos dois fatores denominados legitimação do abuso pela sedução infantil e legitimação pela ausência de violência. De forma específica, esta pesquisa possui como escopo buscar evidências de validade fatorial e consistência interna da escala para o contexto brasileiro, considerando a necessidade de adotar medidas válidas para essa temática no Brasil. Para isso, foram realizados dois estudos, o primeiro para validação exploratória da escala e o segundo para sua validação confirmatória, descritos a seguir.

Estudo 1: Validação Exploratória da Escala de Crenças sobre o Abuso Sexual (ECAS)

\section{Método}

\section{Participantes}

Tratou-se de uma amostra de conveniência, não probabilística, composta por 208 estudantes de graduação de universidades de ensino superior da cidade de João Pessoa, no estado da Paraíba (Brasil), com idades entre 16 e 54 anos $(M=21,64 ; D P=4,90)$, em maior parte do sexo feminino $(65,9 \% ; N=137)$. Essa amostra foi distribuída entre estudantes de Direito $(50 \%, N=$ 104) e de Psicologia (50\%, $N=104)$, provenientes de universidades públicas e privadas. A escolha por essa amostra justifica-se por serem representantes de cursos de futuros profissionais que atuarão na acolhida, tratamento e/ou coleta de depoimento das vítimas de abuso sexual infantojuvenil, dando direcionamentos às condutas acerca desse fenômeno na área forense como, por exemplo, o depoimento especial. 


\section{Instrumentos}

Foram utilizados como instrumentos neste estudo: um questionário sociodemográfico, a título de caracterização da amostra e a Escala de Crenças sobre o Abuso Sexual (ECAS) (Machado, Gonçalves, \& Matos, 2000).

Anteriormente à coleta de dados, foi necessário adaptar o instrumento do português de Portugal para o português do Brasil. Diante disso, realizou-se um estudo piloto com 10 estudantes universitários seguindo a amostra principal, a fim de constatar a compreensão dos itens, das instruções e da escala de respostas. Os critérios para a permanência dos itens era haver concordância com relação à redação e à compreensão dos itens acima de $80 \%$ entre todos os participantes do piloto. Houve boa compreensão da redação e da escala e sua formatação de resposta, tendo apenas a necessidade de substituição do termo "rapariga" por "menina".

Dessa forma, o instrumento permaneceu com os 17 itens da escala original, com opções de resposta em uma escala Likert de 5 pontos que varia de 1 (Discordo totalmente) a 5 (Concordo totalmente). A escala original possui três fatores: legitimação do abuso pela sedução infantil (itens: 4, 6, 8, 9 11, 12, 13 e 16); legitimação pela ausência de violência (itens: 7, 9, 10, 11, 13, 14, 15 e 17); e a crença de que o abuso não existe quando o ofensor não corresponde a um estereótipo pré-definido (itens: 1, 2, 3, 4 e 5) (Ladeiro, 2014). Contudo, os estudos anteriormente realizados consideraram a escala como uma medida genérica, unifatorial, cuja a consistência interna dos itens apontou um alfa de Cronbach 0,80 e 0,84 considerados satisfatórios (Fazenda, 2010; Querido, 2010)

\section{Procedimentos Éticos e de Coleta de Dados}

Os instrumentos foram administrados coletivamente nas salas de aula por pesquisadores previamente treinados, e foram respeitados todos os aspectos éticos, incluindo autorização institucional, o Termo de Consentimento Livre e Esclarecido firmado por cada um dos universitários participantes e o de assentimento para os menores de idade.

O projeto de pesquisa foi aprovado pelo Comitê de Ética em Pesquisa do Centro de Ciências da Saúde da Universidade - CEP/CCS (Prot. $\mathrm{n}^{\circ}$ 0516/15. CAAE: 49371915.1.0000.5188). Esta pesquisa atendeu às determinações da Resolução 466/12, do Conselho Nacional de Saúde, que traça as diretrizes e normas reguladoras de pesquisas envolvendo seres humanos.

\section{Procedimento de Análise dos Dados}

Os dados foram tabulados e analisados por meio do Pacote Estatístico para Ciências Sociais (SPSS) para Windows versão 21.0. Realizaram-se análises fatoriais exploratórias por intermédio do método dos componentes principais com o intuito de conhecer a estrutura fatorial da escala. Utilizou-se também o alfa de Cronbach ( $\alpha$ de Cronbach) para verificação da consistência interna da escala.

\section{Resultados e Discussões}

Inicialmente, procurou-se comprovar a fatorabilidade da matriz de correlações entre os itens da escala; os resultados apoiaram a adequação de se utilizar uma análise fatorial exploratória, observados os seguintes índices que se mostraram adequados: $\mathrm{KMO}=0,897 \mathrm{e}$ o Teste de Esfericidade de Bartlett, $X_{(136)}^{2}=1130,283$; $p<0,000$.

Vale ressaltar que, na primeira análise, foram fixados três fatores conforme proposta dos estudos iniciais da escala; contudo, os resultados não foram satisfatórios com itens duplicados nos fatores e carência de interpretabilidade. Em um segundo momento, realizouse a Análise dos Componentes Principais (ACP) sem fixar, a priori, o número de componentes a extrair, com a rotação oblimim. Dessa forma, foram identificados quatro componentes com valores próprios superiores a 1 (critério de Kaiser), explicando 56,8\% da variância total, com os seguintes valores (eigenvalues): 6,101; 1,302; 1,186 e 1,067, sendo bem distante a diferença de eigenvalue do primeiro fator para os demais. Usando o critério de Cattell (scree plot), observou-se uma estrutura fatorial de um componente.

Com o objetivo de dirimir quaisquer dúvidas acerca dessa estrutura unifatorial, realizou-se uma análise paralela (AP) de Horn (1965), que é um critério mais robusto para retenção de fatores (Hayton, Allen, \& Scarpello, 2004), com o banco de dados ( $N$ $=208$ ) considerando os 17 itens da escala e $95 \%$ de confiança. Verificaram-se, pois, os seguintes valores próprios na análise de Horn: 1,54; 1,42; 1,34 e 1,26, sendo comparados com os anteriormente encontrados, os resultados também sugerem uma estrutura unifatorial. Com base nesses critérios que apontavam para uma estrutura unifatorial da escala e na tentativa de uma análise mais parcimoniosa, optou-se pela realização de uma nova análise fatorial exploratória com o método dos componentes principais, fixando-se apenas um fator e rotação oblimin. 
O ponto de saturação utilizado para o item fazer parte do fator foi de 0,40 (Hair, Anderson, Tatham, \& Black, 2016). Os itens 1 (É muito raro uma criança/ adolescente ser abusada sexualmente) e 3 (As pessoas que abusam sexualmente de crianças/adolescentes são quase sempre desconhecidas) ficaram com as cargas fatoriais abaixo do ponto de corte e com as comunalidades também baixas, sendo item 1 (carga fatorial 0,36; comunalidade 0,13); item 3 (carga fatorial 0,34; comunalidade 0,18). Os demais itens da escala obtiveram cargas variando de 0,48 a 0,72 e a comunalidade de 0,23 a 0,52. O alfa de Cronbach de 0,872, mas pontuando aumento do alfa em caso de eliminação dos dois itens anteriormente citados. Com a eliminação dos itens 1 e 3, ficou a escala final com 15 itens, com cargas fatoriais variando de 0,42 a 0,72 e alfa de Cronbach de 0,88 . Os resultados da análise exploratória são apresentados na Tabela 1.

Diante dos resultados expostos na Tabela 1, pode-se verificar que a Escala de Crenças sobre Abuso Sexual (ECAS) apresenta um índice de consistência interna satisfatório, constatando-se a identificação de um único fator, considerando uma pontuação geral da escala. Altas pontuações nessa escala para os estudos de Fazenda (2010) e Querido (2010) são indicativos de maior tolerância com algo que é ilícito, no caso, o abuso sexual infantojuvenil, propondo que o índice total da escala corresponde a uma medida genérica

Tabela 1.

Estrutura Fatorial da Escala de Crencas Sobre o Abuso Sexual (ECAS)

\begin{tabular}{|c|c|c|}
\hline Itens & $\begin{array}{l}\text { Cargas } \\
\text { Fatoriais }\end{array}$ & $\mathrm{h}^{2}$ \\
\hline 1. É muito raro uma criança/adolescente ser abusada sexualmente. & ---- & 0,13 \\
\hline 2. Só as meninas são vítimas de abuso sexual. & 0,42 & 0,18 \\
\hline $\begin{array}{l}\text { 3. As pessoas que abusam sexualmente de crianças/adolescentes são quase sempre } \\
\text { desconhecidas. }\end{array}$ & ----- & 0,11 \\
\hline 4. A maioria das queixas de abuso sexual são fantasias da criança/adolescente. & 0,57 & 0,34 \\
\hline 5. Os abusadores são pessoas que parecem diferentes das pessoas normais. & 0,48 & 0,23 \\
\hline 6. As crianças podem provocar o abuso pelo seu comportamento sedutor. & 0,64 & 0,41 \\
\hline 7. Só se pode falar em abuso quando há violência. & 0,68 & 0,47 \\
\hline $\begin{array}{l}\text { 8. A maioria das queixas de abuso são inventadas pelas mães (por exemplo: nos casos } \\
\text { de divórcio). }\end{array}$ & 0,60 & 0,36 \\
\hline 9. Se uma menina já foi abusada antes, então é natural que outros homens abusem dela. & 0,51 & 0,26 \\
\hline $\begin{array}{l}\text { 10. Se alguém abusa sexualmente de uma criança/ adolescente é porque não consegue } \\
\text { arranjar parceiras(os) adultas(os). }\end{array}$ & 0,61 & 0,37 \\
\hline $\begin{array}{l}\text { 11. Se uma criança/adolescente só se queixa do abuso muito mais tarde, então ele } \\
\text { provavelmente não existiu. }\end{array}$ & 0,68 & 0,47 \\
\hline 12. As adolescentes levam homens mais velhos a abusar delas. & 0,70 & 0,49 \\
\hline $\begin{array}{l}\text { 13. Só com crianças pequenas é que se pode falar de abuso. Se um(a) adolescente se } \\
\text { envolve com alguém mais velho, a culpa é sua. }\end{array}$ & 0,65 & 0,42 \\
\hline 14. Se o abusador não tiver magoado a criança/ adolescente, o abuso é pouco grave. & 0,65 & 0,42 \\
\hline 15. Só se pode falar de abuso se a criança/adolescente resistir fisicamente. & 0,70 & 0,49 \\
\hline 16. A maioria das queixas de abuso sexual são falsa. & 0,68 & 0,46 \\
\hline 17. Se não houver penetração, então o abuso é pouco grave. & 0,72 & 0,52 \\
\hline Número de itens & \multicolumn{2}{|c|}{15} \\
\hline Valor próprio & \multicolumn{2}{|c|}{5,91} \\
\hline Variância explicada (\%) & \multicolumn{2}{|c|}{39,42} \\
\hline Alfa de Cronbach $(\alpha)$ & \multicolumn{2}{|c|}{0,88} \\
\hline
\end{tabular}


de tolerância/legitimação do abuso sexual. Contudo, com base na análise fatorial exploratória do presente estudo e no cálculo do alfa de Cronbach, pode-se concluir que a escala final reúne um total de 15 itens, com um único fator que mensura as crenças acerca do abuso sexual infantojuvenil, o qual apresenta parâmetros psicométricos satisfatórios e adequados para uso no contexto brasileiro.

Entretanto, reconhecendo o caráter essencialmente exploratório da técnica estatística empregada, destaca-se a importância da realização de outra pesquisa para a comprovação fatorial dessa medida. Em função disso, um segundo estudo foi realizado com outros participantes com características demográficas semelhantes ao estudo anterior, com o objetivo de testar a replicabilidade da estrutura unifatorial obtida no Estudo 1, por meio de uma análise confirmatória com uso do AMOS.

Estudo 2: Validação Confirmatória da Escala de Crenças sobre Abuso Sexual

\section{Método}

\section{Participantes}

Como no primeiro estudo, tratou-se de uma amostra de conveniência, não probabilística. Participaram desse segundo estudo 209 estudantes de graduação de instituições de ensino pública e privada da cidade de João Pessoa no estado da Paraíba, Brasil, com idades entre 16 e 50 anos $(M=23,10 ; D P=6,28)$, em maior parte do sexo feminino $(60,3 \%, N=126)$. Com relação aos cursos, optou-se pelos mesmos do primeiro estudo, Direito $(52,2 \%, N=109)$ e Psicologia $(47,8 \%, N=$ 100), de universidades públicas e privadas. Apesar de a amostra apresentar características semelhantes, trata-se de duas distintas.

\section{Instrumentos}

Assim como no Estudo 1, optou-se por fazer uso de um questionário sociodemográfico, a título de caracterização da amostra e da Escala de Crenças sobre o Abuso Sexual (Machado et al., 2000), adaptada e validada no Estudo 1. Para esse estudo, optou-se pela aplicação do instrumento composto por 17 itens com escala de resposta do tipo Likert, variando de (1) Discordo totalmente a (5) Concordo totalmente. Destaca-se aqui que a manutenção dos dois itens que obtiveram carga fatorial inferior a 0,30 no Estudo 1 foi para fins de comprovação de sua retirada ou não da escala final, tendo em vista maior precisão estatística dos estudos confirmatórios.

\section{Análise dos Dados}

Para a efetuação das análises dos dados, foram utilizados os programas estatísticos SPSS (Pacote Estatístico para Ciências Sociais) para Windows versão 21.0, para o cálculo da consistência interna das medidas e análises complementares; e, o programa AMOS, que é um software de modelização de equações estruturais (SEM) que permite a investigação de estudos pela análise multivariada, incluindo regressões, análises fatoriais, correlações e análises da variância, o qual foi empregado para realizar análises fatoriais confirmatórias, tendo em conta a matriz de covariância e adotando o estimador ML (máxima verossimilhança). Os casos omissos (missing data) foram substituídos pela média das respostas obtidas em cada item.

Para conhecer o ajuste do modelo proposto, foram utilizados os seguintes indicadores: a razão do qui-quadrado pelos graus de liberdade $\left(\chi^{2} / g l\right)$, no qual valores menores do que 5 indicam um ajuste adequado do modelo; o goodness of fi tindex (GFI), o adjusted goodness of fit index (AGFI) e o comparative fit index (CFI), no qual valores próximos ou superiores a 0,90 indicam ajuste bom; e o root mean square error of approximation (RMSEA), cujos valores de 0,06 ou menor indicam ajuste satisfatório, aceitando-se aqueles de até 0,10 (Byrne, 2009; Garson, 2012).

\section{Resultados e Discussão}

Levando-se em conta os achados do estudo anterior, realizou-se uma análise fatorial confirmatória, testando-se uma estrutura unifatorial da escala proposta no Estudo 1. Foram testados três modelos, sendo um trifatorial com 17 itens (modelo proposto pelos estudos iniciais da escala); unifatorial com os 17 itens saturando em um único fator geral chamado de Crenças frente ao Abuso Sexual Infantojuvenil (modelo proposto no Estudo 1) e um modelo unifatorial reduzido para 11 itens. Os indicadores de ajuste para os modelos testados podem ser observados na Tabela 2.

Os resultados obtidos na testagem do modelo trifatorial evidenciam valores marginais para aceitação de ajustamento do modelo proposto para mensuração das crenças sobre abuso sexual. No entanto, para que fosse possível a testagem do modelo trifatorial, foi necessário redistribuir itens para que cada item estivesse presente em um único fator, característica ausente na versão 
Tabela 2.

Índices de Ajuste dos Modelos Testados

\begin{tabular}{lcccccc}
\hline & $\chi^{2}(\mathrm{gl})$ & $\chi^{2} / \mathrm{gl}$ & GFI & AGFI & CFI & RMSEA (IC $-90 \%)$ \\
\hline Modelo 1 & $200,74(101)$ & 0,99 & 0,89 & 0,85 & 0,87 & $0,069(0,055-0,083)$ \\
Modelo 2 & $253,90(119)$ & 0,13 & 0,87 & 0,84 & 0,83 & $0,074(0,061-0,086)$ \\
Modelo 3 & $85,12(43)$ & 0,98 & 0,93 & 0,89 & 0,93 & $0,069(0,047-0,090)$ \\
\hline
\end{tabular}

Nota. Modelo 1 = Trifatorial. Modelo 2= Unifatorial com 17 itens. Modelo $3=$ Unifatorial com 11 itens .

original da ECAS, como pode ser constatado na descrição da escala, anteriormente exposta. Machado et al. (2000) distribuíam a escala em três fatores: legitimação do abuso pela sedução infantil (itens 4, 6, 8, 9 11, 12, 13 e 16); legitimação do abuso sexual pela ausência de violência (itens 7, 9, 10, 11, 13, 14, 15 e 17); e a crença de que não há abuso quando o ofensor não corresponde a um estereótipo predefinido (itens 1, 2, 3, 4 e 5) (Ladeiro, 2014). Pode ser observado que os itens 4 , 9, 11 e 13 se repetem em mais de um fator, o que não é aceitável em um estudo de análise fatorial. Realizando uma análise de conteúdo dos itens, eles foram reagrupados de forma que o modelo trifatorial ficou composto com os seguintes fatores e itens correlacionados: a legitimação do abuso pela sedução infantil (itens 4, 6, 8, 9, 11, 12, 13 e 16); b) a sua legitimação pela ausência de violência (itens 7, 10, 14, 15 e 17); e c) a crença de que o abuso não existe quando o ofensor não corresponde a um estereótipo predefinido (itens 1, 2, 3 e 5).

A confiabilidade composta para o fator Legitimação do abuso sexual pela sedução por parte da vítima foi de 0,74 , o que é um valor aceitável; no entanto, devese apontar para os valores de saturação padronizada no fator dos itens 04 e 08 , que se apresentam abaixo da média geral obtida no fator, o que sugere inadequação de representação da dimensão hipotetizada para esses itens. Já a confiabilidade composta para o fator Legitimação pela ausência de violência foi de 0,75 , o que é um valor aceitável; no entanto, deve-se apontar para o valor de saturação padronizada no fator do item 10 , que se apresentam abaixo da média geral obtida no fator, o que sugere inadequação de representação da dimensão hipotetizada para esse item.

A confiabilidade composta para o fator relacionado ao estereótipo do agressor foi de 0,61 , o que é um valor abaixo do adequado para a leitura de confiabilidade do fator; no entanto, aceitável na condição de estudos exploratórios para desenvolvimento de um instrumento (Hair et al, 2016). Ainda se deve apontar para o valor de saturação padronizada do item 01 , que se apresenta abaixo da média geral obtida no fator, o que sugere inadequação de representação da dimensão hipotetizada para este item.

O teste da diferença de $\chi^{2}$ entre o modelo trifatorial e o modelo unifatorial com 17 itens, para verificar se há diferença significativa de qualidade do ajustamento dos modelos testados, foi efetuado. O resultado obtido $\Delta \chi^{2}(\mathbf{1 8})=\mathbf{5 3 , 1 6} ;(p<0,01)$, evidencia uma superioridade estatisticamente significativa para o modelo unifatorial da escala de crenças sobre abuso sexual. No entanto, a presença de itens com baixa saturação no modelo unifatorial apontou para a necessidade da exclusão de alguns itens para melhor adequação da proposta unifatorial.

Após a exclusão de 06 itens, os 02 do Estudo 01 e mais 04 pela análise confirmatória com auxílio do AMOS, avaliou-se que o modelo unifatorial reduzido obteve índices satisfatórios, superiores ao modelo unifatorial inicialmente proposto. Na Figura 1 é apresentada a estrutura fatorial desse modelo reduzido.

$O$ teste da diferença de $\chi^{2}$ entre o modelo unifatorial reduzido e o modelo trifatorial, para verificar se há diferença significativa de qualidade do ajustamento dos modelos testados, foi efetuado. O resultado obtido, $\Delta \chi^{2}(58)=115,62 ;(p<0,01)$, evidencia uma superioridade estatisticamente significativa para o modelo unifatorial reduzido da escala de crenças sobre abuso sexual. $\mathrm{Na}$ Figura 1, pode-se observar as saturações $(\lambda$, lambdas) que foram estatisticamente diferentes de zero $(\lambda \neq 0 ; z>1,96, p<0,05)$, variando de 0,44 a 0,63 , com uma saturação média de 0,56 .

É possível identificar na Figura 1 que o modelo final da escala reteve 11 dos 17 itens contidos na versão original da escala, pois os itens 01 (É muito raro uma criança/adolescente ser abusada sexualmente), 02 (Só as meninas é que são vítimas de abuso sexual), 03 (As pessoas que abusam sexualmente de crianças/adolescentes são quase sempre desconhecidas), 04 (A maioria das queixas de abuso sexual é fantasia da criança/adolescente), 08 (A maioria das queixas de abuso sexual 


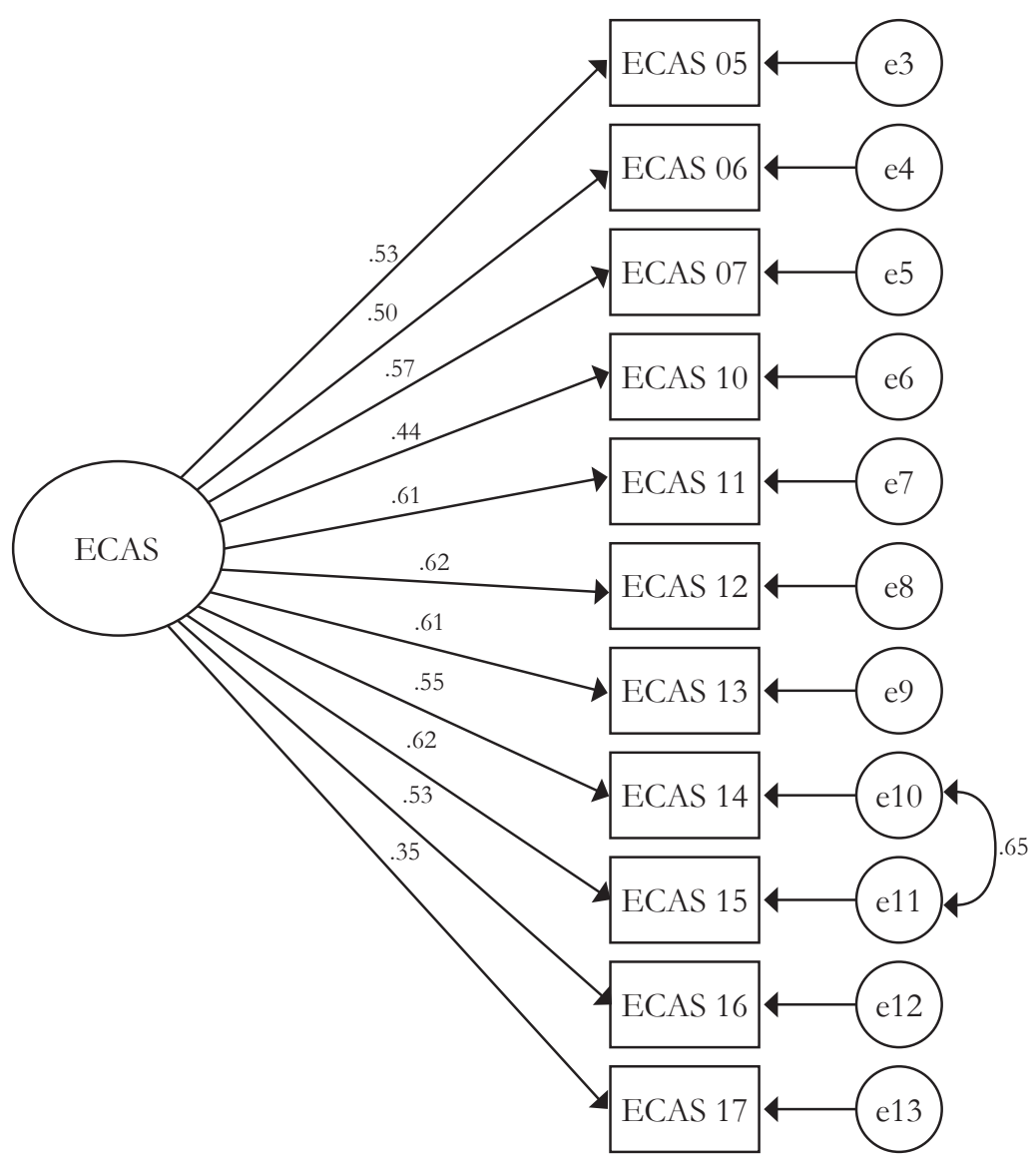

Figura 1. Modelo unifatorial da escala de crenças sobre abuso sexual.

é inventada pelas mães - por exemplo, nos processos de divórcio) e 09 (Se uma menina já foi abusada antes, então é natural que outros homens também abusem dela), apresentaram saturação abaixo de 0,40 , afetando consideravelmente a qualidade do instrumento. Bem como se mostrou necessário, a partir dos índices de modificação, especificar a covariância entre os erros do item 14 (Se o abusador não tiver magoado a criança/ adolescente, o abuso é pouco grave) com o item 15 (Só se pode falar de abuso se a criança/adolescente resistir fisicamente), o que é explicado quando se observa o conteúdo pertinente às covariâncias identificadas. Buscou-se ainda conhecer o índice de consistência interna da medida calculado pelo alfa de Cronbach $(\alpha)$, resultando em um valor de 0,84 .

Foi calculada a confiabilidade composta, indicada para verificação no contexto da análise confirmatória; a partir dessa técnica, obteve-se um valor de 0,84 , corroborando a análise pelo alfa de Cronbach $(\alpha)$, o que sugere adequação dos itens quanto à consistência interna, podendo se inferir também a validade de construto presente na escala unifatorial (Hair et al., 2016; Marôco, 2014). Diante dos resultados apresentados, verificou-se a adequabilidade do modelo unifatorial, corroborando-se o Estudo 01, o qual apresentou indicadores satisfatórios de validade de construto e consistência interna (Pasquali, 2010).

\section{Discussão Geral}

$\mathrm{Na}$ presente pesquisa, os dois estudos apresentados sobre a ECAS mostram evidências satisfatórias de validade fatorial e consistência interna da escala, enfatizando uma medida acerca das crenças frente ao abuso sexual infantojuvenil, uma vez que foi identificada uma estrutura unifatorial, com base em uma validação cruzada, contrapondo a estrutura trifatorial ressaltada nos estudos realizados em Portugal. Os estudos anteriormente realizados com a escala ECAS (Almeida, 2003; Ladeiro, 2014) assumem uma estrutura trifatorial, contudo, não fazem avaliação acerca das consistências internas para cada fator, nem realizam estudos de 
replicabilidade. Destacam também que a soma total do questionário constitui uma medida genérica de tolerância/legitimação do abuso sexual, apontando para uma estrutura unifatorial subjacente, embora os fatores permitam avaliar as crenças centrais subjacentes a tal legitimação. Os estudos de Fazenda (2010) e Querido (2010), que também utilizaram a escala, constataram índices de consistência interna, semelhantes aos aqui encontrados, contudo não fizeram estudos confirmatórios.

$\mathrm{Na}$ escala original, a distribuição dos itens por fator não obedeceu aos critérios estatísticos de que cada item só pode pertencer a único fator, descumprindo um dos pressupostos da análise fatorial que é a estrutura simples de seus componentes (Hair et al., 2016). Em função dessa questão, e dos resultados aqui encontrados, o presente estudo propôs uma mudança com relação à estrutura fatorial, sendo proposta a unifatorialidade da escala e a retirada de 06 itens que foram os de número 1, 2, 3, 4, 8 e 9, ficando a escala final com 11 itens, apresentando um alfa de Cronbach 0,88. A ECAS mensura as crenças frente ao abuso sexual infantojuvenil organizada em torno de um único fator.

Tendo em vista o conceito de crenças como uma representação que atua diretamente nas atitudes e comportamentos, influenciando nas tomadas de decisões conforme destacado por Camino et al. (2013), Maciel et al. (2015) e Salgado (2012), a utilização da ECAS proporciona a compreensão acerca do abuso sexual infantojuvenil, haja vista que conhecer as crenças sobre o fenômeno nos permite compreender as ações e as possíveis decisões tomadas em casos que envolva o tema. Essa escala validada para o contexto brasileiro pode ser uma ferramenta importante para análise das crenças e, consequentemente, comportamentos dos sujeitos que possuem suas práticas e atuações voltadas para educação, acolhimento, suporte, prevenção e manutenção dos direitos e da saúde física e mental da criança e do adolescente.

\section{Considerações Finais}

Entende-se que o abuso sexual infantojuvenil representa uma problemática vigente, dolorosa e relevante, conquistando perceptibilidade e incitando a reprovação e a preocupação com o fenômeno. Porém, essa manifestação social ainda não foi suficiente para libertar a temática do abuso sexual das inibições, dos tabus e das crenças negativas às quais ainda continua entrelaçada. Almeida (2003) afirma que essas crenças, chamadas por ela de mitos, estão disseminados no discurso social, podendo responder com a negação ou minimização do abuso, dificultando a recuperação das vítimas; remontando assim ao papel das crenças na formação das condutas, destacado por Camino et al. (2013).

É nesse contexto que se dá a importância da validação da Escala de Crenças sobre Abuso Sexual Infantojuvenil (ECAS) adaptada para o contexto brasileiro, uma vez que, a partir do conhecimento destas em amostras diversas, podem ser propostas estratégias de combate e prevenção ao fenômeno. A ECAS vem preencher uma lacuna existente em relação a instrumentos voltados a mensurar as crenças sobre o abuso sexual infantojuvenil no Brasil. Nesse sentido, destaca-se que a escala poderá contribuir para área social e científica já que as crenças estão diretamente associadas ao comportamento de proteção, prevenção ou até mesmo violação dos direitos das crianças e dos adolescentes, colaborando para políticas públicas e orientação dos profissionais e da comunidade.

Apesar de a ECAS se apresentar como uma opção adequada para fins de pesquisa, é possível elencar algumas limitações nos estudos realizados, mas que, ainda assim, não inviabilizam a sua importância em estudos da área. Entre as limitações do presente estudo, verifica-se a necessidade de testar a estrutura da escala em outras amostras brasileiras, além da universitária, assim como a validade convergente do construto e a correlação da ECAS com outras variáveis, como a desejabilidade social, haja vista se tratar de um fenômeno que ainda incita muitas polêmicas, as quais podem levar os indivíduos a se comportarem de acordo com as bases normativas da sociedade.

\section{Referências}

Almeida, A. (2003) Abuso sexual de crianças: Crenças sociais e discursos da Psicologia (Dissertação de mestrado em Psicologia da Justiça). Universidade do Minho, Braga. Recuperado de https://repositorium.sdum. uminho.pt/bitstream/ 1822/3197/1/Tese\%20 mestrado\%20Catarina\%20Almeida.pdf

Bustos, P., Rincón, P., \& Aedo, J. (2009). Validación preliminar de la escala infantil de síntomas del trastorno de estrés postraumático en niños/as y adolescentes víctimas de violencia sexual. Psykhe (Santiago), 18(2), 113-126. doi: 10.4067/S071822282009000200008 
Byrne, B. M. (2009). structural equation modeling with AMOS: Basic concepts, applications, and programming. $2^{\mathrm{a}}$ ed. New York: Taylor \& Francis.

Camino, L., Torres, A. R. R., Lima, M. E. O., \& Pereira, M. E. (2013). Psicologia Social: Temas e Teorias. $2^{\mathrm{a}}$ ed., Brasília, DF: Technopolitik.

Eloy, C. B. (2010). A representação social do abuso sexual infantil no contexto judiciário. Revista de psicologia da UNESP. 9(2), 66-78.

Espindola, G. A., \& Batista, V. (2013). Abuso sexual infantojuvenil: A atuação do programa sentinela na cidade de Blumenau/SC. Psicologia: Ciência e Profissão, 33(3), 596-611. Recuperado de http://www. redalyc.org/articulo.oa?id $=282028779007$

Fazenda, I. M. E. M. (2010). Representações sociais em torno do abuso sexual de crianças numa amostra de médicos e enfermeiros dos cuidados de saúde primários. Recuperado de https://estudogeral.sib.uc.pt/jspui/ handle/10316/18219

Florentino, B. R. B. (2015). As possíveis consequências do abuso sexual praticado contra crianças e adolescentes. Fractal: Revista de Psicologia, 27(2), 139-144. doi: $10.22409 /$ frp.v27i2.805

Freire, M. L., \& Alberto, M. D. F. P. (2013). Centro de referência especializada de assistência social: Suporte organizacional para atuação do psicólogo. Cadernos de Psicologia Social do Trabalho, 16(2), 167-182. Recuperado de http://pepsic. bvsalud.org/scielo.php?script $=$ sci_arttext\&pi$\mathrm{d}=$ S1516-37172013000200003

Garbin, C. A. S., Dias, I. D. A., Rovida, T. A. S., \& Garbin, A. J. Í. (2015). Desafios do profissional de saúde na notifıcação da violência: Obrigatoriedade, efetivação e encaminhamento. Ciência \& Saúde Coletiva, 1879-1890. Recuperado de http://www. redalyc.org/pdf/630/63038653024.pdf

Garson, G. D. (2015). Structural equation modeling. Asheboro, NC: Statistical Associates Publishers.

Hair, Jr., J. F., Black, W. C., Babin, B. J., Anderson, R. E., \& Tatham, R. L. (2016). Multivariate data analysis $\left(7^{\mathrm{a}}\right.$ ed.). Upper Saddle River, NJ: Pearson Education.

Hayton, J. C., Allen, D. G., \& Scarpello, V. (2004). Factor retention decisions in exploratory factor analysis: A tutorial on parallel analysis. Organizational Research Methods, 7, 191-205. doi: 10.1177/1094428104263675
Horn, J. L. (1965). A rationale and test for the number of factors in factor analysis. Psychometrika, 30, 179185. doi: $10.1007 / \mathrm{BF} 02289447$

Ladeiro, C. (2014). Percepção e valoração do diagnóstico de abuso sexual em crianças e adolescentes pelos profissionais de enfermagem. Recuperado de https://repositorio-aberto.up.pt/ bitstream/10216/77713/2/33779.pdf

Lei n. 8.069, de 13 de Julho de 1990. Dispões sobre o Estatuto da Criança e do Adolescente e dá outras providências. Recuperado de http://www.planalto. gov.br/Ccivil_03/leis/L8069Compilado.htm

Lein. 8.072, de 25 de Julho de 1990. Dispõe sobre os crimes hediondos nos termos do art. $5^{\circ}$ da Constituição, inciso XLIII, da Constituição Federal e determina outras providências. Recuperado de http://www. planalto.gov.br/ccivil_03/Leis/L8072.htm

Lei n. 13.431, de 4 de abril de 2017. Estabelece o sistema de garantia de diretos da criança e do adolescente vítima ou testemunha de violência e altera a Lei n. 8.069, de 13 de julho de 1990 (Estatuto Da Criança e do Adolescente). Recuperado de http://www. planalto.gov.br/ccivil_03/_ato2015-2018/2017/ lei/L13431.htm

Lima, J. W. D., Alberto, M. D. F. P., Santos, V. M. D., Brito, K. L., \& Silva, S. L. G. (2014). Violência sexual infantojuvenil: $O$ que dizem os documentos do juizado? Estudos Interdisciplinares em Psicologia, 5(1), 02-24. Recuperado de http://pepsic.bvsalud.org/scielo.php?script $=$ sci_arttext\&pid =S2236-64072014000100002

Machado, C., Gonçalves, R., \& Matos, M. (2000). Escalas para avaliação do enquadramento cultural da violência contra mulheres e crianças. Braga: Universidade do Minho.

Maciel, S. C., Pereira, C. R., de Lima, T. J. S., \& de Souza, L. E. C. (2015). Desenvolvimento e validação da escala de crenças sobre a doença mental. Psychology, 28(3), 463-473. doi: 10.1590/1678-7153.201528305

Maria, S. G. S. (2012). Abusos sexuais de crianças: Mudanças resultantes de uma intervenção preventiva (Tese de doutorado em Psicologia Comunitária). Lisboa. ISPA - Instituto Universitário Ciências Psicológicas, Sociais e da Vida. Recuperado de http://hdl.handle. net/10400.12/1768 
Marôco, J. (2014). Análise estatística com o SPSS Statistics. ReportNumber, Lda.

Martins, S., Machado, C., Abrunhosa, R., \& Manita, C. (2012). Escala de crenças sobre violência sexual (ECVS). Análise Psicológica, 30(1-2), 177-191. Recuperado de http://www. scielo.mec.pt/scielo.php?script=sci_arttext\&pid $=$ S0870-82312012000100014 \&lng $=$ pt\&tlng $=\mathrm{em}$

Nicoletti, M., Giacomozzi, A. I., \& Cabral, M. F. (2017). Análisis de dos estudios de casos de abuso sexual cometidos por madres. Revista de Psicología (PUCP), 35(2), 423-452. doi: 10.18800/psico.201702.002

Organização Mundial de Saúde. (2014). Relatório mundial sobre a prevenção da violência. Recuperado de http://apps.who.int/iris/bitstre am/10665/145086/5/9789241564793_por.pdf

Organização Mundial de Saúde. (2016). Maltrato infantil. Nota descritiva. Recuperado de http://www.who. int/mediacentre/factsheets/fs150/es/

Pasquali, L. (2010). Testes referentes a constructo: Teoria e modelo de construção. Em L. Pasquali (Eds.). Instrumentaşão psicológica: fundamento e práticas (pp. 165-198). Porto Alegre: Artmed.

Pereda, N., Guilera, G., Forns, M., \& Gómez-Benito, J. (2009). The prevalence of child sexual abuse in community and student samples: A meta-analysis. Clinical psychology review, 29(4), 328-338. doi: 10.1016/j.cpr.2009.02.007

Pincolini, A. M. F., \& Hutz, C. S. (2014). Abusadores sexuais adultos e adolescentes no sul do Brasil: Pesquisa em denúncias e sentenças judiciais. Temas em psicologia, Ribeirão Preto, 22(2). doi: 10.9788/ TP2014.2-03

Platt, V. B., Back, I. D. C., Hauschild, D. B., \& Guedert, J. M. (2018). Violência sexual contra crianças: Autores, vítimas e consequências. Ciência \& Saúde Coletiva, 23, 1019-1031. doi: 10.1590/1413-81232018234.11362016

Querido, A. (2010) Representações sociais em torno do Abuso Sexual de Crianças (Dissertação de mestrado em Ciências da Educação). Coimbra. Faculdade de Psicologia e Ciências de Educação. Recuperado de http://hdl.handle.net/10316/18219

Rossetti, M. O. (2012). Inventário de comportamentos sexuais da criança: Adaptação brasileira e análise de evidências de validade (Dissertação de mestrado).
Instituto de Psicologia, São Paulo. Recuperado de http://pepsic.bvsalud.org/scielo.php? script $=$ sci_ arttext\&pid $=$ S1415-711X2014000100014\&lng $=$ pt $\& \mathrm{t} \operatorname{lng}=\mathrm{pt}$

Sá, C. M. Q., de Lima, E. I. S., Ramos, F. M. C., da Silva, F. E. B., Oliveira, R. S., \& de Araújo Carrilho, C. (2017). Atenção da equipe de enfermagem frente à violência sexual contra crianças e adolescentes. Mostra Interdisciplinar do curso de Enfermagem, 2(2). Recuperado de http:/ / publicacoesacademicas.fcrs. edu.br/index.php/mice/article/view/1142/918

Salgado, F. S. (2012). Crenças de gestores escolares em relação ao bullying (Dissertação de mestrado). Programa de pós-graduação em Psicologia da Universidade Federal de Juiz de Fora. Juiz de Fora, Minas Gerais, Brasil.

Salvagni, E. P., \& Wagner, M. B. (2006). Estudo de caso-controle para desenvolver e estimar a validade discriminante de um questionário de avaliação de abuso sexual em crianças. Jornal de Pediatria, 82(6), 431-436. doi:10.2223/JPED.1523

Santana, S. S., Santana, J. P., \& Lopes R. L. M. (2011). Violência sexual contra crianças e adolescentes: Análise de notificações dos conselhos tutelares e departamento de polícia técnica. Revista Baiana de Saúde Pública, 35, 68. Recuperado de http:// inseer.ibict.br/rbsp/index.php/rbsp/article/ viewFile/148/143

Santos, R. D. C. F. D. (2011). Violência Sexual e a formação de educadores: uma proposta de intervenção. Dissertação de Mestrado, Universidade Estadual Paulista, Presidente Prudente, São Paulo, Brasil. Recuperado de http://hdl.handle.net/11449/92264. Acesso em 08.03.2019

Santos-Iglesias, P., Sierra, J. C., \& Vallejo-Medina, P. (2013). Propiedades psicométricas del index of spouse abuse en una muestra de varones españoles. Terapia psicológica, 31(2), 209-217. doi: 10.4067/ S0718-48082013000200007

Scarpati, A. S., Guerra, V. M., \& Duarte, C. N. B. (2014). Adaptação da escala de aceitação dos mitos de estupro: Evidências de validade. Avaliação Psicológica, 13(1), pp. 57-65. Recuperado de http://pepsic.bvsalud.org/scielo.php?script $=$ sci_ arttext\&pid=S1677-04712014000100008\&lng $=$ pt $\& \operatorname{lng}=\mathrm{pt}$ 
Shackel, A. (2008). The beliefs commonly held by adults about children s behavioral responses to sexual victimization. Child Abuse and Neglect, 32(4), 485-495.

Silva, L. M. P., Ferriani, M. G. C., Beserra, M. A., Roque, E. M. S. T., \& Carlos, D. M. (2013). A escuta de crianças e adolescentes nos processos de crimes sexuais. Ciência \& Saúde Coletiva, 18(8), 2285-2294. Recuperado de http://sociales.redalyc.org/articulo.oa?id $=63027994012$

Soares, E. M. R., da Silva, N. L. L., de Matos, M. A. S., Araújo, E. T. H., da Silva, L. D. S. R., \& Lago, E. C. (2016). Perfil da violência sexual contra crianças e adolescentes. Revista Interdisciplinar, 9(1), 87-96. Recuperado de https://revistainterdisciplinar. uninovafapi.edu.br/index.php/revinter/article/ view/754/pdf_288

Stoltenborgh, M., Van Ijzendoorn, M. H., Euser, E. M., \& Bakermans-Kranenburg, M. J. (2011). A global perspective on child sexual abuse: Meta-analysis of prevalence around the world. Child maltreatment, 16(2), 79-101. doi: 10.1177/1077559511403920

Von Hohendorff, J., Koller, S. H., \& Habigzang, L. F. (2015). Psicoterapia para crianças e adolescentes vítimas de violência sexual no sistema público: Panorama e alternativas de atendimento. Psicologia Ciência e Profissão, 35(1). Recuperado de http:// www.redalyc.org/html/2820/282038428014/

Recebido em: 04/07/2017

Reformulado em: 12/03/2018

Aprovado em: 30/07/2018 
Sobre os autores:

Camila de Alencar Pereira é graduada em Psicologia pelo Centro Universitário de João Pessoa - UNIPÊ, especialista em Avaliação Psicológica pelo IPOG, mestre em Psicologia Social pela Universidade Federal da Paraíba (UFPB), atualmente é psicóloga do Centro Jean Laplanche - Psicanálise, professora da Faculdade Maurício de Nassau - João Pessoa, doutoranda em Psicologia Social pela UFPB e está vinculada ao GPSMDQ- UFPB.

ORCID: 0000-0002-8136-5399

E-mail: camila_alencarpereira@hotmail.com

Silvana Carneiro Maciel é graduada em Psicologia pela Universidade Federal da Paraíba/UFPB (1993), mestre em Psicologia Social pela UFPB (1997), doutora em Psicologia Social Integrado pela UFPB/UFRN- (2007), pós-doutora em Psicologia Social pelo ICS-Portugal (2014), professora associada II da UFPB da graduação e da Pós-Graduação em Psicologia Social, coordenadora do Grupo de Pesquisa em Saúde Mental e Dependência Química. Linha de Pesquisa Psicologia Social da Saúde e bolsista Produtividade em Pesquisa, nível 2.

ORCID: 0000-0003-1489-1126

E-mail: silcamaciel@gmail.com

Camila Cristina Vasconcelos Dias é graduada em Psicologia pela Universidade Federal da Paraíba (2014) e mestre em Psicologia Social pela mesma instituição (2017). Atualmente é aluna do doutorado no Programa de Pós-graduação em Psicologia Social da UFPB, está vinculada ao Grupo de Pesquisa em Saúde Mental e Dependência Química (GPSMDQ-UFPB) como pesquisadora colaboradora atuando em pesquisas relacionadas à saúde mental e às representações sociais.

ORCID: 0000-0001-9359-3201

E-mail: camilacvdias@gmail.com

Tátia Mirellis de Oliveira Alexandre é graduada em Psicologia pelo Centro Universitário de João Pessoa (UNIPÊ) e mestra em Psicologia Social pela Universidade Federal da Paraíba (UFPB). Atualmente, atua como psicóloga na casa de acolhimento para crianças e adolescentes da cidade de Patos-PB e possui interesse por estudos na área de desenvolvimento infantil, políticas públicas, Psicologia clínica e Psicanálise.

ORCID: 0000-0001-9765-1632

E-mail: tatia.mirellis@hotmail.com

Marcelo Xavier de Oliveira tem formação em Psicologia pela Universidade Federal da Paraíba (UFPB), especialista em Saúde Coletiva, mestre em Psicologia (Psicologia Social) pela UFPB, doutor em Psicologia Social pela mesma instituição, atuando principalmente nas seguintes áreas: estudo de representações sociais, comportamento desviante e medidas psicológicas. Interesse em análise de dados a partir de tratamentos quantitativos e qualitativos.

ORCID: 0000-0002-8751-6426

E-mail:mar_celoxoliveira@yahoo.com.br

Carlos Eduardo Pimentel é doutor em Psicologia Social, do Trabalho e das Organizações (PSTO) pela Universidade de Brasília (UnB, 2012), mestre em Psicologia (Psicologia Social, UFPB, 2004), com formação em Psicologia (UFPB, 2005) e Licenciatura em Psicologia pela Universidade Federal da Paraíba (UFPB, 2001). Atualmente é professor adjunto III do Departamento de Psicologia e da Pós-Graduação em Psicologia Social da Universidade Federal da Paraíba.

ORCID: 0000-0003-3894-5790

E-mail: carlosepimentel@bol.com.br

Contato com os autores:

Universidade Federal da Paraíba. Campus I

Rua Cidade Universitária, S/N - Bairro Castelo Branco III

João Pessoa-PB, Brasil

CEP: 58-051-085

Departamento de Psicologia-CCHLA

E-mail: grupopesquisasmdq@gmail.com 